\title{
Potential of bacterial cometabolism as another means of antibiotic decomposition in a wastewater treatment facility
}

\author{
Akihiko Terada ${ }^{1}$ \\ Published online: 25 October 2019 \\ ๑) Springer-Verlag GmbH Germany, part of Springer Nature 2019
}

As it is well recognized, the concern for widespread antimicrobial resistance (AMR) has received substantial attention. According to the report by the review on Antimicrobial Resistance (O'Neill 2016), AMR will claim 10 million people in 2050, meaning that by then the death toll by AMR will exceed that by cancer. Given that the emergence of AMR is derived from the overuse of antibiotics in our lives and in industries such as pharmaceuticals and livestock, the limited or appropriate use of antibiotics would be favorable. Nevertheless, it is not so easy to reduce the amount of antibiotic use because antibiotics have been utilized not only for fighting against infections but also as the preventive treatment and for promoting the growth of livestock-which means a lot of stakeholders are involved. Multiple actions are required to manage such an emerging issue.

Wastewater treatment facilities could be a key site to deter diffusion of AMR to water environments because such facilities receive municipal and industrial wastewaters via a sewer system. As previously summarized from a technological point of view (Wang et al. 2019), state-of-the-art technologies to decompose pharmaceuticals, including antibiotics, are available by photocatalysis, ultraviolet exposure, and so on. These technologies have displayed excellent removals of antibiotics. Despite a promising function in addition to antibiotic degradation, e.g., production of hydrogen (Wang et al. 2018), a common challenge lies in reducing cost burdens for the installation and operation. Every wastewater treatment facility cannot afford to install such an advanced technology capable of combatting AMR.

How can we tackle the spread of antimicrobial resistance genes and the emergence of antibiotic-resistant bacteria with this limited ability in wastewater treatment facilities? Most wastewater treatment facilities are short on budget to invest

Akihiko Terada

akte@cc.tuat.ac.jp

1 Department of Chemical Engineering, Tokyo University of Agriculture and Technology, Koganei, Japan in a new system for the degradation of antibiotics. It seems that indigenous bacteria in a biological unit of a wastewater treatment facility may embrace a potential. Above all, microbial guilds responsible for aerobic ammonia oxidation, a.k.a. ammonia-oxidizing bacteria (AOB), could be responsible for the mitigation of widespread AMR. It has long been noticed that nitrification activity by AOB is positively correlated with the decomposition of micropollutants, including antibiotics (Tran et al. 2009 and Men et al. 2017). The direct evidence on biotransformation of micropollutants by a pure culture of ammonia-oxidizing bacterium was provided, demonstrating that ammonia monooxygenase (AMO) possessed by the bacterium degrades micropollutants as cometabolism of ammonia oxidation (Yu et al. 2018). In addition to the biotransformation of micropollutants, the product of ammonia oxidation by AMO, i.e., hydroxylamine, abiotically degrades micropollutants under aerobic conditions ( $\mathrm{Yu}$ et al. 2018). The combination of biotic and abiotic mechanisms by AOB may facilitate the removal of antibiotics.

When it comes to biological treatment, there is a common and critical downside. In general, degradation rates of micropollutants are much slower than physiochemical treatments. Furthermore, biochemical transformation cannot completely mineralize antibiotics in many cases, raising an issue of the remaining intermediates. The effect of the intermediates on the emergence of AMR is likely a future investigation. On the other hand, the creation of environmental niches, where $\mathrm{AOB}$ can exert cometabolism to the maximum extent, is of importance. A bioprocess-based investigation is also necessary to harness cometabolism of bacteria. Last but not least, when confronting AMRs in wastewater treatment facilities, we need to showcase repertoires of both physicochemical and biological processes effectively degrading antibiotics, depending on any situations we may encounter. Further intensive studies are necessitated to slow down the spread of AMR in natural environments. 


\section{References}

Men Y, Achermann S, Helbling DE, Johnson DR, Fenner K (2017) Relative contribution of ammonia oxidizing bacteria and other members of nitrifying activated sludge communities to micropollutant biotransformation. Water Res 109:217-226

O'Neill J (2016) Tackling drug-resistant infections globally: final report and recommendations. Rev Antimicrob Resist. http://amrreview.org/sites/default/files/160525_Finalpaper_withcover.pdf Accessed 6 Oct 2019

Tran NH, Urase T, Kusakabe O (2009) The characteristics of enriched nitrifier culture in the degradation of selected pharmaceutically active compounds. J Hazard Mater 171(1-3):1051-1057

Wang H, Wu Y, Feng M, Tu W, Xiao T, Xiong T, Ang H, Yuan X, Chew JW (2018) Visible-light-driven removal of tetracycline antibiotics and reclamation of hydrogen energy from natural water matrices and wastewater by polymeric carbon nitride foam. Water Res 144:215-225

Wang J, Zhuan R, Chu L (2019) The occurrence, distribution and degradation of antibiotics by ionizing radiation: an overview. Sci Total Environ 646:1385-1397

Yu Y, Han P, Zhou L-J, Li Z, Wagner M, Men Y (2018) Ammonia monooxygenase-mediated cometabolic biotransformation and hydroxylamine-mediated abiotic transformation of micropollutants in an AOB/NOB coculture. Environ Sci Technol 52(16):9196-9205.

Publisher's Note Springer Nature remains neutral with regard to jurisdictional claims in published maps and institutional affiliations. 